ARTÍCULO DE INVESTIGACIÓN

\title{
ELEMENTOS QUE CONFIGURAN LA PERCEPCIÓN DE SEGURIDAD ESCOLAR EN ESTUDIANTES DE SECUNDARIA
}

\author{
ELEMENTS THAT CONFIGURE SCHOOL SAFETY PERCEPTION IN \\ HIGH SCHOOL STUDENTS

\begin{abstract}
Mercedes Gabriela OrozCo SOlís ${ }^{1}$, Bertha Alicia COlunga RodríGueZ², HÉCTOR RUBÉN BRAVO 3 ANDRADE, NORMA ALICIA RUVALCABA ROMERO ${ }^{4}$, CLAUDia LiLIANA VÁZQUEZ JUÁREZ ${ }^{5}$ Y MARÍA ÚRSULA PALOS TOSCANO ${ }^{6}$ UNIVERSIDAD DE GUADALAJARA, ESCUELA NORMAL SUPERIOR DE JALISCO, ESCUELA NORMAL SUPERIOR DE ESPECIALIDADES
\end{abstract} \\ FECHA RECEPCIÓN: 24/10/2019 - FECHA ACEPTACIÓN: 1/3/2020 \\ Para citar este artículo: Orozco, M., Colunga, B., Bravo, H., Ruvalcaba, N., Vázquez, C., \& Palos, M. (2020). Elementos que \\ configuran la percepción de seguridad escolar en estudiantes de secundaria. Psychologia, 14(2), 85-97 doi: 10.21500/19002386.4376
}

\section{Resumen}

El objetivo de este trabajo fue describir los elementos que configuran la percepción de seguridad escolar en estudiantes de secundaria. Se presenta un estudio cualitativo con enfoque sistémico ecológico. Por medio de un muestreo teórico, se incluyeron a 22 estudiantes, 50 \% hombres con 12 a 16 años, quienes participaron en un grupo focal, previo consentimiento informado de los padres. Los datos recabados fueron audio grabados; se transcribieron y analizaron con el método de análisis de contenido por medio del programa Maxqda 18. Se identificaron 4 categorías que configuran la percepción de seguridad escolar: 1) condiciones de la escuela (físicas y organizacionales); 2) elementos relacionados con los maestros; 3) elementos relacionados con los compañeros, y 4) elementos relacionados con los padres. Los hallazgos sugieren que las condiciones físicas y organizacionales mantienen un papel importante en la con-

1 Departamento de Clínicas de Salud Mental, Centro Universitario de Ciencias de la Salud, Universidad de Guadalajara. Correo electrónico: gabriela. orozco@academicos.udg

2 Escuela Normal Superior de Jalisco. Correo electrónico: bertha.colunga@ensj.edu.mx

3 Departamento de Clínicas de Salud Mental, Centro Universitario de Ciencias de la Salud, Universidad de Guadalajara. Correo electrónico: ruben. bravo.a@academicos.udg.mx

4 Departamento de Psicología Aplicada, Centro Universitario de Ciencias de la Salud -Correo electrónico: nruval@hotmail.com

5 Departamento de Justicia Alternativa, Ciencias Forenses y Disciplinas afines al Derecho, Centro Universitario Tonalá, Universidad de Guadalajara. Correo electrónico: claudia.vazquezjuarez@ensj.edu.mx

6 Escuela Normal Superior de Especialidades- Correo electrónico: rsulapalos@hotmail.com 
figuración del sentido de seguridad escolar. Asimismo, la cercanía y apoyo recibido de actores del contexto (maestros, padres y alumnos) promueve una percepción más positiva de la seguridad.

Palabras clave: Percepción de seguridad escolar, adolescentes, investigación cualitativa, grupo focal.

\section{Abstract}

The aim of this study was to describe the individual elements that configure the school safety perception in middle school students. We designed a qualitative study, with a systemic and ecological approach. Using a theoretical sampling, we included 22 students, 50\% man with an age from 12 to 16 years old, who participated in a focus group, previous informed consent from the parents. The session was videotaped, the data was transcribed and analyzed using content analysis with the program MAXQDA 18. We identified four categories of factors that configure the student's school safety perception: 1) school conditions (physical and organizational); 2) elements related to teachers; 3) elements related to peers; and 4) elements related to parents. The findings suggest that the school's physical and organizational conditions have an important role in the configuration of the school safety perception. Likewise, the closeness and support received by actors from the school context (teachers, parents and peers) favor a more positive perception of school safety.

Key words: School safety, perceptions, adolescents, qualitative research, focus group

\section{Introducción}

La percepción de la seguridad escolar hace referencia al grado en que el alumno se siente seguro de ir y estar en la escuela, considerando que, dentro del entorno escolar, su bienestar físico y socioemocional no se verá afectado (López et al., 2017). En la adolescencia, los sentimientos de seguridad y confianza generados en la escuela influyen en aspectos como la percepción del apoyo de los adultos, la búsqueda de ayuda en casos de victimización y el grado de conectividad con la escuela (Côté-Lussier \& Fitzpatrick, 2016; Lenzi et al., 2017; Aldridge et al., 2015). Por el contrario, la percepción de inseguridad en la escuela se asocia con el incremento de conductas de riesgo, de acoso escolar, bajo desempeño y con la inasistencia a clases (Gower, McMorris \& Eisenberg, 2015; Hidalgo \& Hidalgo, 2015; Hughes, Gaines \& Pryor, 2015). Los estudiantes que se sienten seguros en la escuela tienen más posibilidades de establecer conexiones positivas, promoviendo un mayor sentido de bienestar (Côté-Lussier \& Fitzpatrick, 2016).

Desde una perspectiva sistémica ecológica, el sentido de seguridad escolar se deriva de la interacción bidireccional que mantiene el individuo con los sistemas donde se encuentra inserto (escuela, familia, amigos) y de la manera en que estos sistemas interactúan con otros de mayor orden (Bronfenbrenner, 1979). Las características de estos sistemas influyen en la percepción del sujeto sobre diferentes aspectos del ambiente, donde la parte subjetiva adquiere un papel importante para identificar factores que afectan el sentido de seguridad en la escuela (Lindstrom-Johnson, Burke \& Gielen, 2012).

Las características físicas de la escuela y su entorno son componentes que se considera influyen en la percepción sobre la seguridad escolar (Lindstrom-Johnson, Burke \& Gielen, 2012). Autores como Bosworth, Ford y Hernandaz (2011) reportaron la relevancia que tienen las condiciones físicas, como la presencia de cámaras, bardas perimetrales y puertas en los baños. Perumean y Sutton (2013) señalan que el tamaño de la escuela y el número de alumnos por grupo influye en la percepción de la seguridad escolar. Asimismo, la comunidad donde se ubica la escuela y se realizan los trayectos casa-escuela, predicen el sentido de seguridad escolar (López et al., 2017).

Por otra parte, los comportamientos relacionados con violencia (Morales \& Silva, 2016; Treviño, 2017), portación de armas, consumo de drogas o peleas físicas son otros factores que influyen negativamente en la percepción de seguridad escolar (Mooij, Smeets \& Wit, 2011). Diversos estudios señalan que situaciones como el acoso escolar afectan esta percepción de seguridad, tanto de los estudiantes involucrados como de toda la co- 
munidad educativa (Vidourek, Woodson \& King, 2017; Williams, Schneider, Wornell \& Langhinrichsen-Rohling, 2018). También la violencia de maestro a alumno afecta negativamente, lo que genera la victimización entre pares (López et al., 2017).

En contraste, otros estudios reportan factores protectores que favorecen la percepción positiva, señalando la organización escolar y la conexión con otros como elementos importantes (Lenzi et al., 2017; López et al., 2017). En organización, destaca la importancia que tienen la disciplina, el orden y la existencia de sistemas de reglas claras, así como la presencia de adultos que conocen los procedimientos normativos y están disponibles para aplicarlos (Bosworth, Ford \& Hernandaz, 2011; Fisher, Viano, Curran, Pearman \& Gardella, 2018; Williams et al., 2018). Fisher et al. (2018) mencionan que los ambientes escolares percibidos como autoritarios y estructurados favorecen una percepción más positiva de la seguridad, probablemente debido a que promueven una menor exposición a la violencia.

Otro factor importante que influye en la seguridad escolar es la relación de los estudiantes con los adultos en la escuela (Côté-Lussier \& Fitzpatrick, 2016; Lenzi et al., 2017). La conexión del alumno con la escuela y las personas es uno de los principales factores para el éxito académico y desarrollo socioemocional (Ricard \& Pelletier, 2016). Diversos trabajos coinciden al mencionar que, cuando el estudiante confía en sus maestros, percibiéndolos como disponibles y dispuestos a apoyarle, la percepción de la seguridad escolar aumenta, al igual que el sentido de pertenencia y conexión con la escuela (Côté-Lussier \& Fitzpatrick, 2016; López, et al. 2017; Williams, et al. 2018).

A pesar de la importancia reflejada en las investigaciones presentadas anteriormente, es notoria la escasez de estudios sobre la percepción de la seguridad escolar realizados en contextos latinoamericanos. Asimismo, la mayor parte de las investigaciones en este tema son estudios cuantitativos, lo cual impide comprender los componentes subjetivos de este fenómeno. Al hablar de seguridad escolar resulta importante acceder a los elementos que se construyen desde la subjetividad de los individuos si se considera que es a través de la experiencia que el sujeto comprende e interactúa con su entorno. Por lo tanto, el objetivo de este trabajo es describir los elemen- tos que configuran la percepción de seguridad escolar en estudiantes de secundaria.

\section{Método}

El diseño de este estudio se basa en el paradigma de investigación cualitativa, asumiendo una perspectiva fenomenológica que se centra en la comprensión de la estructura esencial de la experiencia que vive el individuo (Barbera \& Inciarte, 2012). Desde esta postura descriptiva, comprensiva e interaccionista, se parte de la premisa de que se puede acceder a la subjetividad a partir de lo que dicen y hacen las personas en un escenario sociocultural.

\section{Participantes}

La muestra estuvo constituida por 22 estudiantes de una secundaria pública, la mitad hombres, con edades entre 12 a 16 años, seleccionados por medio de muestreo teórico. Cabe señalar que la escuela se encuentra ubicada en una colonia clasificada con un nivel de marginación alto (Instituto de Información Estadística y Geográfica de Jalisco, 2010). Los participantes fueron divididos en dos grupos focales, con por lo menos tres integrantes de cada grado escolar en cada grupo a entrevistar. Con la finalidad de preservar el anonimato de los participantes, se les asignó una letra del alfabeto para identificarlos.

\section{Técnicas para la obtención de la información}

Para la recolección de datos se implementó la técnica del grupo focal, según Hamui-Sutton y Varela-Ruiz (2013), este espacio suele traducirse en espacio idóneo para propiciar la comunicación y explorar pensamientos entre las personas en un ambiente de interacción y apertura, facilitando la discusión eficiente y plural de actitudes, vivencias y creencias de los informantes.

\section{Procedimientos}

La reunión con el director de la escuela fue el punto de partida de la investigación; le fue presentado el proyecto por parte del investigador principal, planteándole el objetivo del estudio y las actividades que se reali- 
zarían una vez obtenidos los permisos correspondientes. Como parte de la logística diseñada en el protocolo de investigación, se integraron los dos turnos escolares para identificar posibles variaciones en las percepciones. La selección de participantes fue apoyada por un docente en cada turno escolar, reclutando al azar a los estudiantes. Se integraron dos grupos focales de diez estudiantes cada uno, se registraron datos de los estudiantes y se envió un formato impreso de consentimiento a los padres para recabar su firma. Las entrevistas a los dos grupos focales se realizaron en el mismo día para ambos turnos, durante el horario regular de clases, con una duración aproximada de 90 minutos cada una. Inicialmente, se proporcionó información general acerca del propósito de la investigación y las principales consideraciones éticas. Las entrevistas grupales fueron realizadas por el investigador principal, auxiliado de dos coinvestigadores capacitados en la técnica de grupos focales y con experiencias previas en investigación cualitativa, y quienes cuidaron el desarrollo logístico de las sesiones. Se utilizó una guía de entrevista semiestructurada. El contenido fue audiograbado. Al finalizar las sesiones, los informantes se reincorporaron a sus actividades escolares. No recibieron remuneración o algún estímulo por su participación.

\section{Análisis de los datos}

Se utilizó análisis de contenido, ya que permite tratar el discurso y diversas acciones humanas a manera de textos que, al ser analizados mediante un sistema de categorías, contribuyen a comprender fenómenos sociales, como la seguridad escolar (Andréu, 2011). Para ello, las entrevistas fueron transcritas por personal capacitado; se realizó la lectura general del contenido textual, en búsqueda de patrones conceptuales y para identificar significados emergentes que los participantes externaron en relación con la seguridad escolar. Durante la lectura inicial se combinaron elementos de los modelos categoriales deductivo e inductivo; la identificación y etiquetación de categorías la realizaron dos investigadores con experiencia en investigación cualitativa. Posteriormente, se generaron unidades de registro para codificar los datos relevantes, según las preguntas de investigación iniciales, además de aspectos emergentes. La incorporación de los registros propició la creación de nuevas categorías, así como el desarrollo de subcategorías, a veces distantes, de lo inicialmente considerado, ya que el discurso de los participantes manifestaba la presencia de otros temas relevantes. Durante todo el proceso se llevó a cabo una triangulación entre el análisis del discurso y la revisión documental. Las entrevistas se transcribieron en el programa Word; una vez procesados, los datos dieron lugar a documentos preliminares que fueron analizados con el programa MAXQDA 18.

\section{Consideraciones éticas}

Como referente ético se tomó lo establecido en el artículo 17 del Reglamento de la Ley General de Salud en Materia de Investigación para la Salud, donde se considera esta investigación como de nivel I, es decir, que no representa un riesgo para los participantes (Secretaría de Salud, 2014).

En el desarrollo del estudio se tomaron en cuenta consideraciones éticas que incluyeron la obtención del asentimiento de los adolescentes participantes y el consentimiento informado de sus padres. Previo al inicio de la sesión, se les comentó a los estudiantes que su participación sería voluntaria y confidencial. Asimismo, se les indicó que los resultados globales de la investigación serían reportados a las autoridades escolares en un informe técnico, el cual no incluiría ningún elemento que revelara su identidad, buscando promover el diseño de estrategias para prevenir posibles riesgos a los que se encuentren expuestos.

\section{Resultados}

Se identificaron cuatro categorías: 1) condiciones de la escuela que influyen en la seguridad, incluyendo tres subcategorías que abordan las características físicas, organizacionales y académicas; 2) elementos relacionados con los maestros, con tres subcategorías que engloban aspectos del apoyo académico, socioemocional y lo referente al manejo de conflictos; 3) elementos relacionados con los pares, y 4) elementos relacionados con los padres. En los dos últimos casos se encontraron dos subcategorías que abordan elementos del apoyo académico y socioemocional.

\section{Condiciones de la escuela}




\subsection{Características físicas y seguridad}

El mantenimiento y cuidado de las bardas perimetrales fueron referidos por los informantes, quienes tienen presentes acciones como fortalecer las bardas, aumentar su altura y tapar hoyos. Asimismo, hacen referencia a que, a pesar de que en algunos casos los maestros pueden saber sobre condiciones de riesgo existentes en el entorno físico, como los hoyos, desde su experiencia no han realizado acciones para corregirlas: »(...) en diferentes hoyos que han abierto los mismos delincuentes o alumnos de aquí para hacerse la pinta y que los maestros todavía no saben, o que los maestros sí saben, pero todavía no hacen algo para tapar esos hoyos« (Participante $\mathrm{M})$.

La presencia de situaciones relacionadas con violencia, tanto en los alrededores como dentro de la escuela, se perciben como negativas y algo que se debe evitar para no meterse en problemas. Desde lo mencionado por los participantes, se encontró que se percibe la presencia de conductas de riesgo, como el uso de sustancias, el portar armas y las agresiones relacionadas con pandillas. Sin embargo, los mismos estudiantes refieren no reportar estas situaciones a las autoridades educativas, argumentando que es mejor evitar los espacios donde suceden: »(...) pues yo no me voy para allá y aquí pos, pasan drogas por las rejas de las canchas, se arriman y dan drogas, cigarros, cuchillos, armas... han llegado a recoger pistolas, solo que no digo nada« (Participante $\mathrm{M}$ )

\subsection{Características organizacionales y de normatividad}

En diferentes momentos del discurso resalta la importancia de las reglas y su implementación, identificándolas como elementos clave para conservar la disciplina y seguridad dentro de la escuela.

Pues en cada salón hay como un reglamento. Están en todas las aulas (...) de vez en cuando los maestros dicen: "Oye, iléeme el número sabe que!, y iya!” Y itú nomás lo lees!... algunos alumnos que de vez en cuando sí hacen desorden, saben el reglamento y las sanciones... como que sí dicen, ay mejor no... iPero sí sirven las reglas! (Participante I).

Desde lo referido por los participantes, uno de los aspectos fundamentales para mejorar la seguridad en la escuela es la aplicación consistente de las reglas, ta- rea que es considerada como responsabilidad exclusiva de los maestros. Los participantes mencionan que exigir un mejor comportamiento a los alumnos podría prevenir conductas de riesgo que afectan a todos, como las drogas o el abandonar la escuela.

\subsection{Características académicas}

Se encontró una relación cercana entre la percepción del desempeño y la seguridad emocional. Particularmente, situaciones emergentes, como los exámenes, representan momentos que se experimentan como altamente influyentes en el estado emocional, sobre todo con relación a las posibles consecuencias que el estudiante percibe:

Supongamos que cuando no tenemos nada que hacer vamos acá bien relajados, bien chirindongo, pero cuando tenemos exámenes (...) uno como que se empieza a tensar y dice: "no, imagínate... si me equivoco en esto"; pues ya todo salió mal y es la presión que tiene uno ahí (Participante $\mathrm{O}$ ).

Los aspectos académicos relacionados con el desempeño y el desarrollo educativo son los principales factores estresores mencionados por los participantes en distintos momentos. Se identifica una percepción general de que el desempeño actual tiene una influencia directa en el poder continuar con su formación académica, situación que se vive como preocupante, y, por lo tanto, se considera que influye en la seguridad emocional.

\section{Elementos relacionados con los maestros}

\subsection{Apoyo académico del docente}

Los profesores se perciben como preocupados por el desempeño académico de los estudiantes y dispuestos a apoyarles en sus actividades. Algunos ejemplos de las acciones que mencionaron les ayudan a sentirse más seguros en el área académica son: la disponibilidad del maestro para resolver dudas, el uso de distintos métodos de enseñanza y el dedicar tiempo a poner atención al estado emocional:

Yo opino que algunos maestros se salen de la rutina y... no es tanto el explicar, sino que nos sintamos bien al seguir aprendiendo. Pero a la vez convivir, digo (...) el maestro ve 
cómo estamos y nosotros nos vamos cono-

ciendo (Participante $\mathrm{K}$ ).

Un punto que se considera disminuye el sentido de seguridad y justicia en el aula es la percepción de que existan tratos especiales para algún estudiante, siendo denominados como »privilegios«. Se encuentra, entonces, que percibir que hay estudiantes que no realizan los mismos trabajos o hacen el mismo esfuerzo, pero que obtienen calificaciones aceptables, ocasiona molestia e inconformidad:

A mí me ha pasado que, en el salón, una chica que no entrega trabajos, isiempre le ponen 8 o 9!, y la verdad, a mí me molesta. ¡Porque a mí me cuesta trabajo y me esfuerzo mucho para hacerlo!, para que una niña, que no hace nada, le regalen calificación que no merece (Participante D).

\subsection{Apoyo socioemocional del docente}

El apoyo socioemocional que se recibe del docente fue relacionado con la seguridad emocional del estudiante desde distintos aspectos. A pesar de que se mencionaron otros actores, como las personas encargadas del aseo, el maestro es percibido como una influencia central sobre qué tan seguro se siente un alumno en la escuela. Desde la experiencia de los estudiantes, hay diferentes acciones que el docente hace en la cotidianidad y que favorecen el sentido de seguridad socioemocional de los estudiantes, destacando la disponibilidad para brindar contención emocional:

(...) los maestros, cuando me ven triste, me preguntan: ¿oye qué tienes? ¿Estás bien? ¿Te puedo ayudar en algo? Y si te preguntan, te dan una sonrisa y te dicen: »si ocupas algo dime y si quieres llorar, aquí estoy, se va a quedar entre nosotros, pero quiero verte bien«, o sea, sí hay apoyo (Participante J).

Asimismo, el que un docente se encuentre atento al estado emocional de los estudiantes y se involucre activamente para mejorarlo, son elementos que aparecen como importantes para el sentido de seguridad en la escuela:

Pues que los profesores cuando nos ven, por ejemplo, llegamos a la primera hora y nos ven como desanimados, nos dicen, por ejemplo: »ila siguiente clase los quiero ver más alegres!« (...) es como que el maestro va de buenas y nos quiere ver muy bien en las clases (Participante F).

Aunado a lo anterior, el intervenir en los conflictos que el alumno puede tener con sus pares es otro elemento que influye en el sentimiento de seguridad y confianza. Otras acciones del docente que se experimentan como positivas para la seguridad emocional en la escuela incluyen el tomar en cuenta los intereses de los estudiantes al interactuar con ellos y centrar los procesos educativos en aspectos que llamen su atención: »(...) Mis compañeros tienen más confianza con el maestro (...) él nos habla más como jóvenes, y no como nada más estudiar, estudiar, estudiar" (Participante $\mathrm{H}$ ).

Finalmente, permitir que los estudiantes se brinden contención entre ellos mismos, cuando es necesario, es otro de los elementos que se rescatan como promotores de sentimientos de seguridad y confianza: »Y la maestra no nos hace por separarnos, no, se espera un momento a que nos apoyemos a esa persona y, luego ya después, nos dice que cada quien en su lugar $(. .$.$) «Par-$ ticipante $\mathrm{H}$ ).

\subsection{Docente y conflicto}

Otra subcategoría identificada fue el papel que desempeña el maestro en la aplicación de la disciplina y el abordaje de los conflictos. Se encuentra entonces que sentir que no hay un adulto dispuesto a intervenir de manera constante en las situaciones de riesgo es un factor que influye negativamente en la percepción de seguridad escolar.

(...) yo diría que eso no tiene un alto porque los maestros no ejercen las reglas (...) ahí está el reglamento, pero son como quince reglas y la mayoría no las cumple (...) están golpeándose y el maestro está acá platicando, con otro asunto (...) no se da cuenta hasta que ya ocurre el desastre (Participante M).

\section{Elementos relacionados con los pares}

Los pares representan otra importante fuente de apoyo y soporte para los adolescentes. Al igual como sucedió con los maestros, se evidenció que el apoyo que se brinda en el área académica y socioemocional es el 
principal componente de la relación con los pares que influyen en la percepción de la seguridad.

\subsection{Apoyo académico de los pares}

En el área académica, los participantes rescatan la importancia de apoyar a los pares que tienen dificultades para comprender materias o realizar cierto tipo de actividades. En el contenido del discurso se observa la aparición del término "compañerismo", haciendo referencia a ayudarse mutuamente a sobrepasar las demandas académicas a las que se puedan enfrentar en sus actividades escolares. En este sentido, la reciprocidad es un elemento importante del apoyo académico entre pares; se valora como una herramienta que permite tener certeza de que en un futuro recibirán el mismo apoyo que se está brindado al otro.

Sería como el compañerismo (...) alguien no le entiende, va y me dice que si le puedo ayudar, y ya sería como que te ayudo, pero sabes que si yo algún día yo necesito ayuda, también, algún día tendrás que ayudarme (Participante $\mathrm{H})$.

\subsection{Apoyo socioemocional de los pares}

A diferencia de como ocurrió con los docentes, los pares aparecen como una fuente de preocupación e inseguridad, siendo pocas las experiencias de apoyo que se encontraron en el rubro socioemocional.

La convivencia, siempre va a afectar tanto en lo académico como en lo emocional (...) no de buena manera, la verdad es de mala, es que raro. iRarísimo!, que sea de buena manera (...) se reúnen, pero para cosas malas, para hacerle bromas al maestro (...) (Participante J).

Las principales experiencias de apoyo socioemocional de los pares se viven al interior de grupos más reducidos de amigos, donde se refiere un sentido de lealtad que deriva del tiempo de amistad. Es a partir de estas interacciones donde los participantes mencionan sentirse apoyados, sobre todo en el sentido de reciprocidad del que se hablaba en el área académica.

Pues yo siento que cuando son más amigos, yo siento que te pueden ayudar más, ¿no?, o sea, que ya como ya llevas más años conociéndolos, te ayudan más, y como otros que no te llevas tanto o casi no les hablas o así, pues sientes que no te pueden ayudar (Participante T).

Con respecto a los pares con los que no se mantiene una relación de amistad, la relevancia proporcionada a la lealtad parece favorecer una cierta resistencia a interactuar con miembros de otros grupos. Lo anterior se puede expresar con actitudes de respeto y mantenimiento de la distancia en algunos casos, pero de agresión y discriminación en otros:

(...) Los rechazan porque a veces quiere (...) uno que siempre se quiere juntar, pero lo rechazan (...) Una vez uno le quiso ayudar, pero le dijeron que no (...) que se va a hacer del otro lado (...) como que lo rechazan por eso porque algunos le quieren ayudar, pero por miedo no, por miedo a que lo discriminen también (Participante Q).

\section{Elementos relacionados con los padres}

Finalmente, se encuentra que la participación de los padres en aspectos relacionados con la escuela es otro elemento que influye con el sentido de seguridad de los estudiantes, identificando dos subcategorías que incluyen aspectos académicos y socioemocionales. El percibir que los padres se interesan en el desempeño académico y el comportamiento favorece estados emocionales positivos y promueve mayor interés a la escuela, así como una orientación más positiva a la misma: »Yo creo que la autoestima cambia, (...) si los padres se preocupan, se interesan por mí y este... te esfuerzas más cuando se interesan más, en lo que hago (...)» (Participante D).

\subsection{Apoyo académico de los padres}

Con relación a lo académico, un aspecto que se consideró importante para la seguridad escolar fue el apoyo que los padres brindan para que el estudiante cumpla con sus tareas y tenga un desempeño académico exitoso. El sentir que los padres buscan opciones para ayudarles promueve un sentido de apoyo que favorece una orientación más positiva a las tareas académicas:

Pues yo pienso que el apoyo es fundamental, (...) por decir así en Inglés (...) a veces que tu mamá no entendió inglés o que no le tocó estudiarlo, hay veces que, pues, 
se preocupa y anda buscando con primos o tíos o el que sabe para poderte ayudar a ti (Participante H).

Asimismo, se consideró como positiva la participación de los padres para proveer a la escuela de recursos extra, ya sea para mantenimiento de las instalaciones o para el desarrollo de eventos extraescolares. El percibir que los padres se mantienen en conexión con la escuela es un aspecto que incrementa el sentimiento de apoyo y bienestar de los estudiantes:

(...) los padres participan por lo regular en la escuela, tienen, por ejemplo, "lo de tecnología« (...) cuando la escuela necesita dinero para el próximo año que vienen los de primero, (...) que tengan el equipo que ya dañaron las generaciones anteriores (...) a recaudar fondos para que los alumnos se sientan bien y puedan tener el apoyo (...) (Participante I).

\subsection{Apoyo socioemocional de los padres}

La familia es un entorno que se considera influye en el estado emocional de manera importante. De acuerdo con lo encontrando, los participantes consideran que la existencia de conflictos en el hogar puede afectar negativamente el estado emocional del estudiante en la escuela:

(...) yo todo creo que viene desde la casa, si te fue bien en la casa, vas a llegar bien a la escuela, pero si te enojaste con un familiar y llegas a la escuela, vas a estar mal. Tratas de ponerte bien, pero no es lo mismo estar feliz a tratar de ser feliz (Participante O).

La participación de los padres en la escuela, no solo en los aspectos de mantenimiento, sino también en cuanto al seguimiento del comportamiento del estudiante, es percibida como un aspecto importante con relación a la seguridad. Desde la perspectiva de los participantes, cuando los padres monitorean el comportamiento de los alumnos e intervienen oportunamente es posible prevenir que se vean involucrados en problemas: »Creo que es bueno que se preocupen y que vean que estamos bien (...) es para ver si están bien, porque hay veces que, en esta etapa, nos confundimos y nos vamos desviando del 'buen camino' (...) « (Participante J).
Con relación al punto anterior, se rescató la relevancia de que exista una adecuada comunicación familiaescuela, comentando que los maestros suelen preocuparse por que los padres conozcan cuando sus hijos tienen problemas, ya sea en áreas académicas o conductuales. Sin embargo, refieren que, en ocasiones, los propios padres no se interesan por los estudiantes, o son los alumnos quienes evitan que sus padres reciban información sobre su comportamiento. De tal forma, trabajar en mejorar la comunicación entre la escuela y la familia fue un elemento central para favorecer una percepción de mayor seguridad y bienestar en los estudiantes: »(...) y así se sentirían más seguro los alumnos y los padres, para que se sientan unidos los alumnos y a la vez suba su autoestima (...) (Participante G).

\section{Discusión}

El objetivo del presente trabajo fue describir los elementos que configuran la percepción de seguridad escolar en estudiantes de secundaria. Desde lo referido por los participantes, se encuentra que la percepción de la seguridad escolar se ve influida por aspectos relacionados con las características físicas de la escuela y con elementos derivados de las experiencias de los estudiantes en interacción con sus maestros, pares y padres de familia.

Con respecto a los aspectos físicos del entorno escolar, se encontró que los estudiantes consideran que existen condiciones, como la existencia de hoyos en las paredes, que favorecen conductas de riesgo y disminuyen la percepción de la seguridad en la escuela. Este hallazgo coincide con otros estudios donde se reporta que los factores físicos que se relacionan con la protección y mantenimiento del espacio se consideran relevantes al hablar del sentido de seguridad (Bosworth et al., 2011; Díaz-Vicario \& Gairín, 2017). Como lo mencionan Espinoza y Rodríguez (2017), la importancia que tienen los aspectos físicos de la escuela en la generación de ambientes positivos tiene que ver, principalmente, con qué tanto las condiciones físicas favorecen espacios adecuados para la interacción constructiva con otros. De tal manera, es probable que los aspectos físicos mencionados se relacionen con la seguridad escolar debido a la influencia que podrían tener en variables interpersonales, como el seguimiento de las reglas y la protección de los estudiantes. 
Sin embargo, es necesario seguir profundizando en este punto en futuras investigaciones, de manera que se analice la influencia que podría tener el componente interpersonal en la valoración de aspectos físicos y relevantes para la percepción de la seguridad escolar.

En este punto resulta importante destacar que a pesar de que los estudiantes saben sobre estas condiciones de riesgo y las perciben como un aspecto negativo, refieren no reportarlas, depositando en los maestros la responsabilidad de identificarlas y corregirlas. Como lo mencionan Susanne, Pereda y Guilera (2017), las situaciones que implican un riesgo pueden promover una perpetuación de la llamada "cultura del silencio", donde los observadores prefieren no reportar debido al temor de las posibles consecuencias. Por lo tanto, es importante no solo generar estrategias enfocadas en promover en los estudiantes una postura más proactiva en la construcción de un entorno escolar seguro, sino también desarrollar las condiciones para reportar y solucionar los riesgos físicos de manera segura.

La organización escolar y la aplicación de la disciplina fueron otros componentes relacionados con la percepción de la seguridad escolar desde lo referido por los participantes. Principalmente, se observa que la existencia de un sistema de reglas mantiene un papel importante en la percepción de la seguridad escolar, encontrando que el maestro aparece como una entidad central, donde uno de los aspectos más relevantes es el proceso de gestión de la disciplina. Como lo mencionan Bosworth, Ford y Hernandaz (2011), el maestro es un componente fundamental para que el estudiante se sienta en una atmosfera segura, encontrando que la consistencia en la aplicación de acciones que dan un sentido de disciplina y control aumenta la percepción de seguridad.

Con relación al punto anterior, desde la experiencia de los estudiantes, el docente es percibido como poco atento a la implementación de medidas disciplinarias para controlar el comportamiento disruptivo o de desorden de sus alumnos, lo que es diferente en otros aspectos, como el cumplimiento de las tareas o los exámenes de conocimientos. Este resultado coincide con lo mencionado por diversos autores que identifican un debilitamiento de la función normativa en el maestro, siendo percibido como apático o desinteresado en la aplicación de reglas (Conde, 2014; López de Mesa-Melo,
Soto-Godoy, Carvajal-Castillo \& Urrea-Roa, 2013). Esta situación ha sido referida en estudios previos, encontrando como causa de la aparente apatía del profesor el temor que este puede sentir para corregir a los alumnos, derivado del aumento de las situaciones de violencia contra los profesores por parte de los estudiantes y de las reformas en las normativas institucionales, que reducen el margen de acción de los docentes para implementar estrategias correctivas de la disciplina escolar (Conde, 2014; Treviño, 2017). Sin embargo, considerando que, desde la experiencia del estudiante, el maestro aparece como poco hábil e inclusive poco interesado en aplicar las medidas disciplinarias de manera consistente, sería importante que futuros estudios se orienten a explorar la perspectiva que tiene el maestro del proceso de gestión disciplinar, lo cual podría abordarse desde un diseño de investigación acción participativa.

Entre los hallazgos de este trabajo, también se encontraron aspectos positivos de la relación con los profesores que influyen en la seguridad socioemocional, los cuales tienen que ver con el apoyo brindado en aspectos académicos y emocionales. Para los participantes, la mayor parte de los docentes se mantienen interesados en generar las estrategias didácticas y extraescolares necesarias para promover un adecuado desempeño académico. Asimismo, el apoyo emocional recibido de los maestros representa una importante fuente de seguridad partiendo de experiencias simples, como el reconocimiento del valor que tiene el estado emocional del adolescente en el proceso educativo. Diversos trabajos coinciden al señalar que la percepción del apoyo del docente en lo académico y socioemocional mantiene una influencia significativa en la autoestima, bienestar y salud mental de los estudiantes (Strati, Schmidt \& Maier, 2017; Tennant et al., 2014; Zamarripa, Castillo, Tomás, Tristán \& Álvarez, 2016). Considerando la relevancia que tiene el apoyo del docente para el bienestar de los estudiantes, las competencias socioemocionales que tiene el maestro para relacionarse con sus alumnos representan un punto clave en la prevención de conductas de riesgo de los adolescentes desde la escuela (Villalobos et al., 2016).

El papel de los pares en la percepción de la seguridad escolar fue un tema que también se exploró, identificando una percepción global negativa sobre las relaciones interpersonales entre compañeros, la cual mantiene 
una influencia desfavorable en el sentido de seguridad en la escuela. Sin embargo, también se encontró que los participantes refieren la existencia de grupos más cerrados de compañeros, considerados amigos, dentro de los cuales les es más sencillo encontrar apoyo académico y socioemocional, pero que, a su vez, generan acciones de exclusión hacia otros alumnos que no pertenecen a dichos grupos. Así también, descata la importancia del apoyo recibido de los pares desde la experiencia referida por los participantes, orientada más hacia el apoyo que brindan a sus compañeros, haciendo énfasis en que ayudar a otro aumenta su sentido de seguridad en la escuela, al percibirse esto como una acción implícita y recíproca que garantiza que, si ayudan, serán apoyados cuando lo requieran en un futuro.

Lo anterior puede ser explicado desde lo mencionado por Castellanos y Zayas (2019), quienes comentan que las relaciones entre pares en la adolescencia se caracterizan por una necesidad de alcanzar un sentido de influencia mutua y reciprocidad en el apoyo. Esta característica favorece que los pequeños grupos se estructuren de manera poco flexible, manteniendo fuertes sentidos de lealtad, lo cual promueve la segregación de los que se encuentran fuera y aumenta el riesgo de situaciones de violencia entre estos pequeños grupos al percibirse como antagónicos. Diversos estudios concuerdan en que la percepción de un bajo apoyo de los pares, de acciones de exclusión y de violencia influyen negativamente en la seguridad escolar y en distintos indicadores de ajuste psicosocial (Morales \& Silva, 2016; Treviño, 2017; Vidourek et al., 2017; Williams et al., 2018).

Un hallazgo relevante de este trabajo, distinto a lo reportado en estudios previos, es lo relativo a la importancia otorgada por los informantes a la participación de los padres en la escuela, presentándose como un factor que influye en el sentido de seguridad escolar. Por otra parte, desde la experiencia de los participantes, un elemento que afecta su seguridad en la escuela tiene que ver con la existencia de conflictos en la familia, explicado por la afectación que genera en su estado emocional, el cual influye en qué tan seguros se sienten cuando están en la escuela. Calvo, Verdugo y Amor (2016) mencionan que la disfunción familiar se proyecta en la escuela con comportamientos inapropiados en los adolescentes, afectando su sentido de seguridad y promoviendo la ge- neración de distintas problemáticas. Desde esta perspectiva, la calidad de las relaciones familiares influye en diversas variables que reflejan el ajuste psicosocial de los adolescentes, siendo importante continuar con estudios que exploren su posible relación con la seguridad socioemocional de los adolescentes en la escuela.

Aunado a lo anterior, se encuentra que la percepción de apoyo por parte de los padres, referido como estar al pendiente de su desempeño académico y conductual, así como colaborar en actividades extraescolares, representa una característica que los participantes relacionaron con la seguridad escolar. Percibir que los padres están informados sobre los posibles problemas que pudieran tener en la escuela y realizar actividades que promueven mejores condiciones en la escuela se consideraron elementos que favorecen sentimientos de apoyo y seguridad en el entorno escolar. La colaboración entre familia y escuela representa un eje central para fomentar que el estudiante se sienta seguro y comprometido dentro del entorno escolar (Calvo, Verdugo \& Amor, 2016). Por lo tanto, es necesario desarrollar futuras investigaciones que permitan generar estrategias de intervención, enfocadas en favorecer la generación de relaciones más cercanas y constructivas entre familia y escuela.

En términos generales, la percepción de seguridad escolar de los adolescentes se vincula con una serie de interacciones con las personas y el entorno, resaltando la importancia del apoyo que se percibe de parte de las personas significativas, como son los maestros y los padres. Particularmente, destaca en el discurso de los jóvenes el apoyo emocional que perciben de parte de sus profesores, interpretado por medio de acciones sencillas que demuestran interés en ellos. Si bien las relaciones con los pares aparecieron como elementos que, desde una perspectiva global, influyen negativamente en la percepción de la seguridad escolar, resulta necesario continuar explorando el papel que las dinámicas establecidas al interior de los grupos de adolescentes desempeñan para mantener el sentido de seguridad en la escuela. Asimismo, las condiciones del entorno material también destacan en el discurso de los estudiantes, de lo cual se deduce la importancia que se debe dar al mantenimiento de la infraestructura que, aunado al interés de los adultos, maestros y padres, es fundamental para comprender la percepción de la seguridad escolar de los adolescentes. 
Este estudio presenta como fortaleza el dar la voz a los adolescentes para identificar, desde su discurso, experiencias que refieren elementos desde los cuáles se configuran sus percepciones de seguridad escolar, que contribuyen a la comprensión del fenómeno psicosocial referido; por otro lado, presenta las limitaciones propias que puede tener un estudio cualitativo al no permitir generalizar los hallazgos, de ahí que se sugiere seguir trabajando el tema con distintas poblaciones y métodos, así como bajo diversas perspectivas teóricas.

\section{Referencias}

Aldridge, J., Fraser, B., Fozdar, F., Ala'i, K, Earnest, J., \& Afari, E. (2015). Students' perceptions of school climate as determinants of wellbeing, resilience and identity. Improving schools, 19(1), 1-22, doi: $10.1177 / 1365480215612616$

Andréu, J. (2011). Las técnicas de análisis de contenido: una revisión actualizada. Fundación Centro Estudios Andaluces, Universidad de Granada, 10(2), 1-34. Recuperado de http://public.centrodeestudiosandaluces.es/pdfs/S200103.pdf

Barbera, N., \& Inciarte, A. (2012). Fenomenología y hermenéutica: dos perspectivas para estudiar las ciencias sociales y humanas. Multiciencias, 12(2), 199-205. Recuperado de https://www.redalyc. org/pdf/904/90424216010.pdf

Bosworth, K., Ford, L., \& Hernandaz, D. (2011). School climate factors contributing to student and faculty perceptions of safety in select Arizona schools. $J$ Sch Health, 81(4), 194-201. doi:10.1111/j.17461561.2010.00579.x

Bronfenbrenner, U. (1977) Toward an Experimental Ecology of Human Development. American psychologist, 32, 513-531. Recuperado de https://pdfs.semanticscholar.org/a5f5/a4f4a4545519d84c5c57095b4b$\begin{array}{llllllllllllllll}\text { c } & \text { c } & 6 & 8 & 5 & \text { d } & 7 & \text { d } & \text { d } & 9 & \text {. } & \text { p } & \text { d } & \text { f ? }\end{array}$ ga $=2.206739984 .287964595 .1571759953$ 1481507652.1567786234

Calvo, M., Verdugo, M., \& Amor, A. (2016). La Participación Familiar es un Requisito Imprescindible para una Escuela Inclusiva. Revista latinoamericana de educación inclusiva, 10(1), 99-113. doi:10.4067/ S0718-73782016000100006

Castellanos, L., \& Zayas, F. (2019). La exclusión entre pares: sus implicaciones en la formación de los y las estudiantes. Diálogos sobre educación, 10(19), 1-17. doi:10.32870/dse.v0i19.531

Conde, S. L. (2014). La violencia y la cultura de la calle entran a la escuela: acciones y reacciones. Sinéctica, 42(1), 1-21. Recuperado de http://www.scielo.org. $\mathrm{mx} /$ scielo.php? script $=$ sci_arttext\&pid $=\mathrm{S} 1665$ 109X2014000100002\&lng=es\&tlng=es

Côté-Lussier, C., \& Fitzpatrick, C. (2016). Feelings of Safety at School, Socioemotional Functioning, and Classroom Engagement. Journal of Adolescent Health, 58(5), 543-550. doi:10.1016/j.jadohealth.2016.01.003

Díaz-Vicario, A., \& Gairín, J. (2017). A comprehensive approach to managing school safety: case studies in Catalonia, Spain. Educational Research, 59(1), 89-106. doi:10.1080/00131881.2016.1272430

Espinoza, L., \& Rodríguez, R. (2017). La generación de ambientes de aprendizaje: un análisis de la percepción juvenil. RIDE. Revista Iberoamericana para la Investigación y el Desarrollo Educativo, 7(14), 110132. doi:10.23913/ride.v7i14.276

Fisher, B., Viano, S., Curran, C., Pearman, A., \& Gardella, J. (2018). Students' Feelings of Safety, Exposure to Violence and Victimization, and Authoritative School Climate. Am J Crim Just, 43, 6-25. doi:10.1007/s12103-017-9406-6

Gower, A. L., McMorris, B. J., \& Eisenberg, M. E. (2015). School-level contextual predictors of bullying and harassment experiences among adolescents. Social Science \& Medicine, 147, 47-53. doi:10.1016/j. socscimed.2015.10.036

Hamui-Sutton, A., \& Varela-Ruiz, M. (2013). La técnica de grupos focales. Investigación en Educación Médica, 2(5), 55-60. Recuperado de http://riem.facmed. unam.mx/sites/all/archivos/V2Num01/09_MI_ HAMUI.PDF

Hidalgo, C., \& Hidalgo, A. (2015). Violencia e inseguridad contextual percibida y roles en bullying en escolares mexicanos. Revista Latinoamericana de Ciencias Sociales, Niñez y Juventud, 13(2), 767-779. doi:10.11600/1692715x.13215021214 
Hughes, M. R., Gaines, J. S., \& Pryor, D. W. (2015). Staying away from school: Adolescents who miss school due to feeling unsafe. Youth Violence and Juvenile Justice, 13(3), 270-290. doi:10.1177/1541204014538067

Instituto de Información Estadística y Geográfica de Jalisco (2010). Marco demográfico del Municipio de Tlaquepaque. Recuperado de www.iieg.gob. $\mathrm{mx} /$ PoblacionVivienda/marcodemografico/M.xls

Lenzi, M., Sharkey, J., Furlong, M., Mayworm, A., Hunnicutt, K., \& Vieno, A. (2017). School Sense of Community, Teacher Support, and Students' School Safety Perception. American Journal of Community Psychology, 60, 527-537. doi:10.1002/ ajcp. 12174

Lindstrom-Johnson, S., Burke, J., \& Gielen, A. (2012). Urban students' perceptions of the school environment's influence on school violence. Children and Schools, 34(2), 92-102. doi:10.1093/cs/ cds016

López de Mesa-Melo, C., Soto-Godoy, M., CarvajalCastillo, C., \& Urrea-Roa, P. (2013). Factores asociados a la convivencia escolar en adolescentes. Educación y Educadores, 16(3), 383-410. Recuperado de https://www.redalyc.org/articulo. oa? id $=834 / 83429830001$

López, V., Torres-Vallejos, J., Villalobos-Parada, B., Gilreath, T. D., Ascorra, P., Bilbao, M., ... Carrasco, C. (2017). School and community factors involved in chilean students' perception of school safety. Psychology in the Schools, 54(9), 991-1003. doi: 10.1002/pits.22069

MAXQDA (Versión 18) [Software de computación]. Berlín: VERBI GmbH

Mooij, T., Smeets, E., \& Wit, W. D. (2011). Multilevel aspects of social cohesion of secondary schools and pupils' feelings of safety. The British journal of educational psychology, 81(3), 369-90. doi:10.1348/000709910X526614

Morales, L., \& Silva, D. (2016). Representaciones sociales de los estudiantes con relación a la violencia entre pares. Revista Aletheia, 8(2), 86-103. Recuperado de http://www.scielo.org.co/pdf/aleth/ v8n2/v8n2a05.pdf
Perumean-Chaney, S. E., \& Sutton, L. M. (2013). Students and Perceived School Safety: The Impact of School Security Measures. American Journal of Criminal Justice, 38(4), 570-588. doi:10.1007/ s12103-012-9182-2

Ricard, N. C., \& Pelletier, L. G. (2016). Dropping out of high school: The role of parent and teacher selfdetermination support, reciprocal friend ships and academic motivation. Contemporary Educational Psychology, 44(45), 32-40. doi:10.1016/j.cedpsych.2015.12.003

Secretaría de Salud (2014). Reglamento de la Ley General de Salud en Materia de Investigación para la Salud, título segundo, capítulo I, art.17.

Strati, A. D., Schmidt, J. A., \& Maier, K. (2017). Perceived challenge, teacher support, and teacher obstruction as predictors of student engagement. Journal of Educational Psychology, 109(1), 131-147. doi:10.1037/edu0000108

Sussane, G., Pereda, N., \& Guilera, G. (2017). ¿Contar o callar?: La respuesta de los adolescentes del sistema de justicia juvenil ante las experiencias de exposición indirecta a violencia. Revista Española de Investigación Criminológica, 3(15), 1-23. Recuperado de https://dialnet.unirioja.es/servlet/ articulo? codigo $=6226915$

Tennant, J., Demaray, M., Malecki, C., Terry, M., Clary, M., \& Elzinga, N. (2014). Students' ratings of teacher support and academic and social-emotional well-being. School Psychology Quarterly, 30(4), 494512. doi: $10.1037 / \mathrm{spq} 0000106$

Treviño, E. (2017). Atravesar el riesgo. Los docentes frente a la violencia en Veracruz. Perfiles educativos, 39(158), 20-37. Recuperado de http:// www.scielo.org.mx/scielo.php? script $=$ sci arttext\&pid $=$ S0185-26982017000400020\&lng $=$ es\&tlng $=$ es.

Vidourek, R. A., Woodson, K. D., \& King, K. A. (2017). School Violent Victimization Among Youth: Is There an Association with Perceived School Safety? Violence \& Victims, 32(1), 181-192. doi:10.1891/0886-6708.VV-D-14-00119

Villalobos, B., Carrasco, C., Olavarría, D., Ortiz, S., Oyarzún, D., Ascorra, P., ... Álvarez, J. (2016). Victimización de pares y satisfacción con la vida: la 
influencia del apoyo de profesores y compañeros de la escuela. Psykhe, 25(2), 1-16. doi:10.7764/ psykhe.25.2.861

Williams, S., Schneider, M. Wornell, C., \& Langhinrichsen-Rohling, J. (2018). Student's Perceptions of School Safety: it is Not Just About Being Bullied. The Journal of School Nursing, 34(4) 319-330. doi:10.1177/1059840518761792

Zamarripa, J., Castillo, I., Tomás, I., Tristán, J., \& Álvarez, O. (2016). El papel del profesor en la motivación y la salud mental de los estudiantes de educación física. Salud mental, 39(4), 221-227. doi:10.17711/SM.0185-3325.2016.026 